\title{
Enhanced functional binning for one- and two-point statistics using a posteriori Uncertainty Quantification of LPT data
}

\author{
P. Godbersen ${ }^{1 *}$, A. Schröder ${ }^{1}$ \\ ${ }^{1}$ German Aerospace Center (DLR), Institute of Aerodynamics and Flow Technology, Göttingen, Germany \\ *Philipp.Godbersen@dlr.de
}

\begin{abstract}
In the evaluation of Lagrangian particle tracking (LPT) measurement data the use of spatially binned flow statistics in the form of one, two or multi-point statistics is often an essential step towards better understanding of the measured flow fields. Increasingly there is a focus towards uncertainty quantification of the measurement system however these evaluations are seldom used to directly improve the statistics by directly involving them into the calculation. We present our Functional Binning approach which makes use of such uncertainty information as a core component for the calculation of improved statistics. The improvements towards prior approaches are shown utilizing synthetic data as well as data from a real-world subsonic jet experiment. Beyond the initial formulation for one-point statistics, we show that this approach is readily extended towards two-point statistics and explore more advanced utilizations of uncertainty information for the optimal selection of particle pairs. Furthermore, the benefits of more individualized particle error estimations are investigated and some strategies for archiving such information are investigated.
\end{abstract}

\section{Introduction}

Lagrangian particle tracking enables the accurate measurement of the position, velocity and acceleration of particles moving within fluid flows (Ouellette et al., 2006; Schanz et al., 2016). The scattered nature of the individually tracked particles provides a great advantage over other related measurement techniques such as PIV. Instead of a fixed regular grid of convolution windows, whose size imposes a low pass filtering effect on resolvable structures, particle positions are distributed in a stochastic manner and provide local point measurements. This can be exploited in Eulerian ensemble averaging using spatial binning to realize very fine resolutions, since the bin size is not directly linked to measurement technique and is primarily limited by the amount of available data. This allows a direct trade-off between bin size and convergence of the underlying statistic. One particularity of LPT is that we do not just have the position and velocity of a particle at each timestep but inherent to the processing a continuous track over a certain time range. This provides additional information as the single time steps are not independent but instead temporally linked. Within this paper we will focus on the Shake-The-Box (STB) approach for LPT where the two variants for time-resolved STB (Schanz et al., 2016) and multi-pulse STB (Novara et al., 2019) express this temporal connection in slightly different ways. For time-resolved STB tracks can span up to the entire measurement duration directly providing significant temporal information. The multi-pulse variant necessary for faster flows provides temporal information only for the short bursts typically consisting of four pulses. This variant relies on collecting many bursts that are temporally uncorrelated from each other to build flow statistics. A small amount of temporal information is nevertheless available for each burst and within this paper we will mostly focus on the processing of such multi-pulse data. The presented approaches are generally not limited to multi-pulse STB but adaptable to any LPT measurement that provides access to this temporal data.

The core idea for our Functional Binning approach (Godbersen and Schröder, 2020) to LPT data processing relies on the fact that this additional temporal information is available which is not utilized in other current binning approaches. Instead of just evaluating the position or velocity at a certain point along the short multi-pulse track (at the pulse times or the midpoint) we now consider a continuous functional track over time. This provides an opportunity to increase the convergence speed of spatially binned statistics simply because now more information is considered. The functional approach extracts the maximum amount of available data 


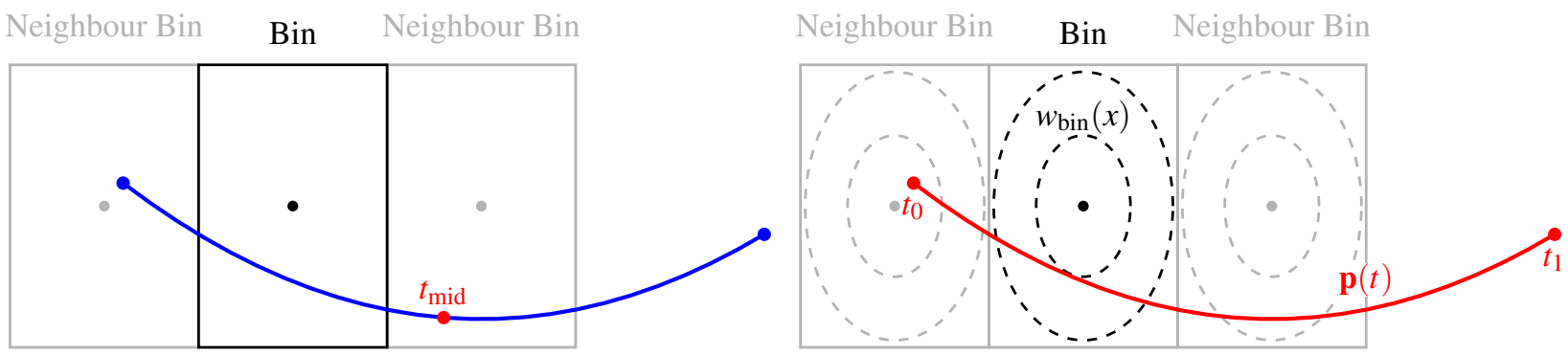

Figure 1: Sketch of convetional binning utilizing the midpoint (left) and the Functional Binning approach (right). Graphics from (Godbersen and Schröder, 2020)

from the tracks provided by the STB processing. Due to the general formulation of the Functional Binning approach, most prior discrete binning strategies can also be expressed within this functional framework (evaluation at midpoint, evaluation at particle positions, spatial weighting strategies, etc.). The approach was initially presented for single point statistics but is readily extended to two-point statistics as well, with the resulting advantages and possibilities being a focus of this paper.

One significant property of Functional Binning is the inherent use of uncertainty information for the calculation of the statistics. Uncertainty quantification of LPT data is of great interest not just for the evaluation of experiment setups but also when processing the resulting data for further statistics. A comprehensive a priori uncertainty estimation trough the full evaluation chain of a LPT measurement is presented by Bhattacharya and Vlachos (2020) allowing for insight into the different error terms contributing to the total measurement error. A different approach is conducted in the strategies by Novara et al. (2016); Gesemann et al. (2016) focusing on an a posteriori uncertainty evaluation starting from a point further along the LPT processing chain (see as well contribution of Gesemann et al. to this ISPIV'21). The approach relies on estimation of the particle position uncertainty and builds the error for all further processing steps by propagating this error instead. For the multi-pulse STB algorithm this approach is utilized to motivate the use of the track midpoint for velocity evaluations as the location of lowest error. The Trackfit approach from Gesemann et al. (2016); Gesemann et al. (2021) applied to time-resolved STB data presents an elegant method to estimate the position accuracy directly from the measurement data and utilizes this knowledge to select optimal smoothing parameters for the track building. Within this paper we will make use of this second approach, utilizing particle position accuracy as a posteriori uncertainty source for further propagation within the functional binning approach

For evaluation of the presented binning approaches we utilize synthetic data using same setup as in the original Functional Binning paper (Godbersen and Schröder, 2020). Synthetic data is useful as it allows full control over the input data and error values that are passed into the processing approaches. The synthetic data used here directly provides tracked particles instead of images that need to be processed by the STB algorithm. We are not directly interested in the performance STB processing itself but want to analyze the postprocessing of such data and need to directly control the particle position error. For an evaluation of the performance of the STB processing in itself we refer the reader to the results of the LPT-challenge (Sciacchitano et al., 2021 ) instead. Synthetic data is attractive because it provides exact ground truth data to judge the resulting statistics against. However, ultimately these post-processing techniques will be applied to real world data so only investigating synthetic data is not satisfying. Therefore we will also present some of these methods applied to a real multi-pulse STB jet experiment (Manovski et al., 2021; Godbersen et al., 2019). As no exact ground truth is available any more, only qualitative comparisons can be made however the improvement in convergence is readily apparent when considering only a subset of the available data.

\section{Functional Binning}

A detailed introduction of the Functional Binning approach can be found in Godbersen and Schröder (2020) but we will provide a short introduction here to provide context for the further developments. Functional Binning relies on the fact that we have a functional expression of the particle position over time available from the STB processing (including TrackFit). For the multi-pulse STB data considered here, tracks take the form of second-order polynomials. Just as we have the tracks in a functional expression, the bins now 
need to be brought into a functional form as well. This takes the form of spatial weighting functions with compact support, defined only in the region we want the bin to be located. In addition to this spatial weight function we also assign a temporal weight function to the track, selecting regions from which we want to extract information from the track. Using these two weighting functions together with the individual track functions we can calculate a continuous weighted average expressed using integrals over the track time:

$$
\mathbf{M}_{\mathbf{V}}=\frac{\sum_{n=1}^{N} \overline{\mathbf{V}}_{n} \bar{W}_{n}}{\sum_{n=1}^{N} \bar{W}_{n}}=\frac{\sum_{n=1}^{N} \int_{t_{0}}^{t_{1}} \mathbf{v}_{n}(t) w_{n}(t) \mathrm{d} t}{\sum_{n=1}^{N} \int_{t_{0}}^{t_{1}} w_{n}(t) \mathrm{d} t} \quad \text { with } \quad w(t)=w_{\text {bin }}(\mathbf{p}(t)) w_{\text {track }}(t),
$$

with the velocity function $\mathbf{v}(t)$ and the weight function $w(t)$ as product of the temporal track weight function and the spatial bin weight function using the track position function $\mathbf{p}(t)$.

This general formulation allows to represent most existing spatial binning strategies by selecting certain functions for the track weight or the bin weight function respectively. The simple binning approach based on cartesian bins can be replicated by selecting a multidimensional top hat function for the bin weight, defined only within the extent of the Cartesian bin. A spatial Gaussian bin weighting strategy for such cartesian bins (Agüí and Jiménez, 1987) can directly be implemented by using a Gaussian bin weight function. A similar consideration can be made for the track weight function, allowing to replicate existing discrete track evaluation schemes within this functional framework. The midpoint approach from Novara et al. (2016) can be directly replicated by considering a Dirac pulse at the midpoint time, selecting just this discrete value in the integration. Similarly, the classic approach of using the four particle positions resulting from the four pulses in time can be replicated by a comb of four such Dirac pulses positioned at the appropriate times (see fig. 2). The functional bin definition allows arbitrary bin definitions far beyond these examples, enabling easy implementation of variable bin sizing, bin orientation and positioning simply by defining an appropriate spatial weighting function for each bin. Adaptive bin shaping could be facilitated based on statistics of an initial evaluation or a curvature based approach as presented by Raiola et al. (2020). One interesting binning concept that is not readily interpreteable in the functional framework is the polynomial fit technique for spatial binning presented in Agüera et al. (2016). The approach improves among other goals the accuracy of statistics in the presence of velocity gradients within the bins. For functional binning this is not as relevant as a main aim here is to allow for higher bin resolutions, thereby reducing the impact of velocity gradients within each individual bin in this manner.

The special cases for the trackweight function mentioned above make apparent where the gain of additional information for the statistics comes from in the functional case. Both of these discrete evaluation schemes waste a lot of available information simply because the weight function is zero for a majority of the track time. For Functional Binning we select a track weight that is non-zero for the entire track time taking into account an uncertainty quantification of the velocity function. The process is further described in the section on uncertainty quantification below.

This approach was initially designed for one-point statistics like mean velocities or Reynolds stresses however some initial extension towards two-point statistics was already presented within the original paper. The functional approach is readily extended to also cover relations between two or multiple particles/tracks. For a two-point correlation on LPT data the distance between particle pairs is relevant for spatial binning as the velocity correlation is performed by spatial binning with respect to the distances of particles to some reference particle at a designated position. Instead of operating on the discrete particles such calculations can also be performed in the functional space. For the multi-pulse data considered here the function space is that of polynomials and many operations are well defined there. Polynomials can be added and subtracted from one another resulting in a new polynomial of the same order. Additionally, multiplication between polynomials is also defined, in this case generally changing the order of the resulting polynomial.

$$
p_{1}(t)+p_{2}(t)=p_{1+2}(t), \quad p_{1}(t)-p_{2}(t)=p_{1-2}(t), \quad p_{1}(t) * p_{2}(t)=p_{1 * 2}(t),
$$

with two polynomials $p_{1}$ and $p_{2}$ and resulting functions also within the polynomial function space. Such operations can be readily expressed in terms of operations on the polynomial coefficients allowing for easy implementation.

Statistics are then expressed as integrals over functions in combination with the temporal and spatial weight functions. For the implementation the goal is to stay as long as possible within this functional space but for the actual final calculation of the statistics we need to return to discretized evaluations of these functions at some point. This is usually done at the stage where we numerically solve the integrals to provide a concrete value to add to the statistic in a bin. Doing the discretization at this late point has the advantage that the whole theory for the numerical calculation of integrals can be relied upon, i.e. the Gauss-Legendre quadrature approach tells exactly where to sample the integrand for a limited number of points while providing certain 


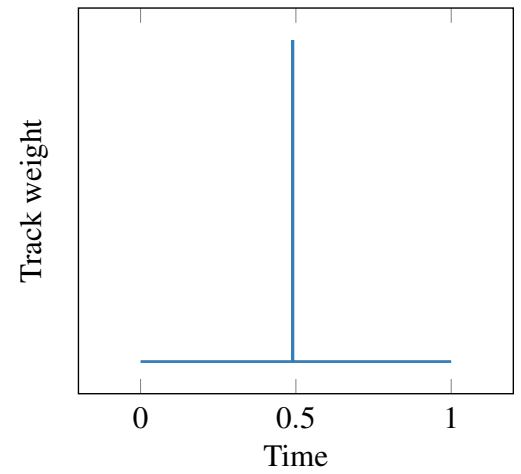

(a) Evaluation at midpoint

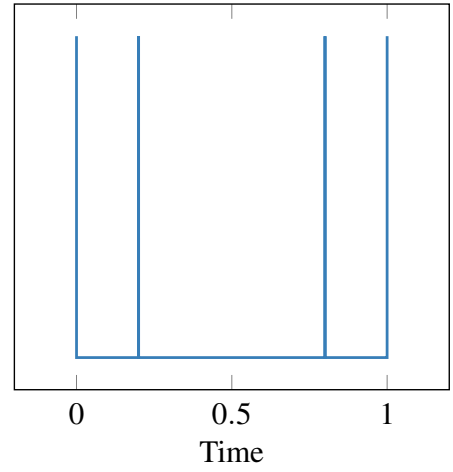

(b) Evaluation at particle positions

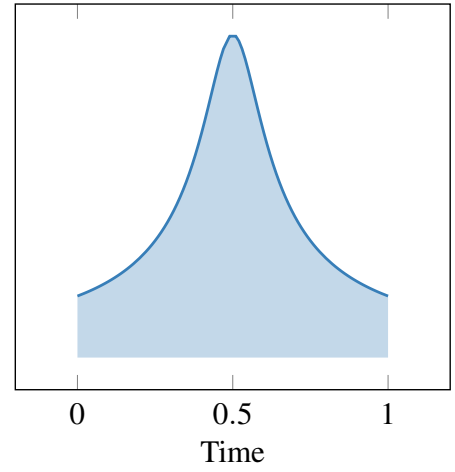

(c) Functional Binning + uncertainty

Figure 2: Different track evaluation schemes expressed as track weight functions of track time within the Functional Binning framework.

optimality guarantees (Bogaert, 2014). Alternatively, for complicated weight functions it might be desirable to utilize an adaptive sampling of the integrand to efficiently concentrate evaluation in regions where more resolution is needed. Again, we can draw upon the well-established methods for numerical integration and could utilize an adaptive Gauss-Kronrod quadrature instead. Doing these calculations in the functional space we can nearly directly attach these two-point statistics to the existing machinery implemented for one-point statistics. A two-point correlation is very similar to the calculation of a velocity mean, except the spatial binning is now done in the relative distance space to some reference track and instead of simply averaging the velocity of a single track, we now consider a mean over the relation of the velocities between tracks.

There is one additional benefit in the calculation of two-point statistics that did not present itself in the original formulation of single-point statistics. As conceptionalized in the initial Functional Binning paper, two-point statistics also benefit from better resolution for close distances. they are generally calculated over a large distance range but the values for close distances are often the most important. One such example is the calculation of Taylor micro-scales based on two-point correlations. For discrete binning approaches these close values are often not very well resolved, especially when utilizing the particle positions directly. This is due to the diffraction limited imaging typically used, where the particle image size is much larger on the cameras than the physical size of the particle itself. As particles get close to one another their images start to overlap on the cameras much earlier than might be expected from their physical size. Such image overlaps then provide a challenge for the LPT processing leading to increased position error or in extreme cases might make them impossible to tell apart. Such concerns do not apply to the midpoint approach as commonly used for multi-pulse STB, but even here benefits are expected as the functional approach simply provides more opportunities for the tracks to get very close to one another. These ideas as presented in Godbersen and Schröder (2020) are used in a recent contribution by Hammond and Meng (2021) to calculate more accurate radial distribution functions for inertial particles.

\section{Uncertainty propagation}

An important element of Functional Binning is the utilization of uncertainty information to design the track weight functions, thereby directly incorporating such knowledge into the calculation of statistics. A constant weight along the track would be feasible, (and sensible for time-resolved STB evaluations barring additional individual knowledge about time-steps), for multi-pulse STB evaluations we know this not to be optimal based on the initial introduction of the method in Novara et al. (2016) The midpoint approach was suggested there directly because of an uncertainty propagation of particle position error trough the tracking scheme and velocity calculation, showing the midpoint to be the location of lowest velocity error. Figure 7(b) shows the result of such an analysis for the velocity error, clearly showing the V-shaped nature of the velocity error with the error increasing towards the edges of the track. The inverse of this curve can directly be used as a track weight for the Functional Binning procedure taking into account that the additional information away from the midpoint is associated with a larger error and should therefore be weighted less when performing the evaluation. 
All uncertainty quantification we consider within this paper relies on the propagation of a posteriori particle position uncertainty trough further processing. It was chosen this way because the raw particle position accuracy can be evaluated from the experiment itself as a "primal" uncertainty of the measurement scheme that then be propagated further along through the processing easily without having to dive too deep into exact uncertainty chain of the concrete measurement technique. This provides an uncertainty "interface" of sorts, making the implementation agnostic to the concrete measurement technique utilized even though this is inspired by the choices made in the two mentioned STB variant developments.

Especially for the time-resolved STB approach this particle position error is readily available from the parametrization of the TrackFit scheme. By calculating the position spectrum of the measured particles, it is possible to identify the noise floor of the measurement in respect to that spectrum which is then translated into a cutoff frequency for an optimal wiener filter for track building. They can then also translate this cutoff frequency into a particle position error value thus providing a direct path for an a posteriori error estimation directly on the concrete measurement data of the experiment which can then be used in further processing.

For multi-pulse STB evaluations this approach is not available due to the missing time resolution across the snapshots. Here we rely on the estimations about typical error values of the method as stated in the introducing paper (Novara et al., 2016) which are supported by results from the critical evaluation of the method in the Pressure Challenge (Van Gent et al., 2017) as well as the recent LPT-Challenge (Sciacchitano et al., 2021). Such results can be verified on the actual measurement data utilized within this paper due to a diagnostic run employed at the start of the experiment campaign. A special run was conducted that captured images from all cameras at the same time Both separate imaging systems should therefore reconstruct the particles at the exact same position and any deviation can be attributed to random positioning error from the processing forming the basis for this approach described by Manovski et al. (2021). As this should be the dominating error for a well calibrated imaging system utilizing Volume Self Calibration (Wieneke, 2008) this allows a pathway to verify these error assumptions for a concrete experiment for a posteriori particle position error estimation. For this experiment this error is within the expected positioning error of $0.1 \mathrm{px}$ (see fig.8(a)). This particle positioning error then forms the basis for further propagation as described above. Linear gaussian error estimation is used to propagate the error through the polynomial fit of the track building stage and the necessary derivation of the polynomial with respect to time to acquire the velocity information or all further derived data.

\section{Application to measurement data}

To provide an overview on the performance the Functional Binning approach is applied to a synthetic dataset with known ground-truth data with velocity values:

$$
u=\frac{\sinh (y)}{\cosh (y)+\varepsilon \cos (x-c t)}+c \quad \text { and } \quad v=\frac{\varepsilon \sin (x-c t)}{\cosh (y)+\varepsilon \cos (x-c t)},
$$

and coeficients $c=3$ and $\varepsilon=0.7$. Synthetic tracks are generated for the spatial domain $\Omega=[-2 \pi, 2 \pi] \times$ $[-\pi, \pi]$ and over the time domain $T=[0,2 \pi]$.

In addition the the synthetic data we also consider the real-world jet measurement. Used here is the jet with the round nozzle, at Mach 0.85 for the measurement domain close to the nozzle. A thorough description of the jet measurement can be found in the referenced publications. Even though a large amount of data is available for this data set, we will show statistics calculated only on a small subset of the available data to make differences in convergence between the methods visually apparent in the profiles. Since no direct ground truth data is available we perform only a qualitative comparison.

Figure 3 shows such comparisons of profiles on small amounts of data for the mean velocity profile of the synthetic data-set. A comparison between axial Reynolds stresses for the jet data set is shown in figure 4. For the synthetic case a strong improvement is immediately apparent. The improvement for the jet data-set is not as drastic but a smoother statistic is visible. This was to be expected as here we are mostly interested in fine profiles along the radial direction to resolve the shear layer. As this direction is orthogonal to the jet axis, and therefore mostly of the track direction, less tracks cross across into neighboring bins in this direction diminishing the effectiveness of the functional approach. This is in contrast to the synthetic experiment where the track movement is within the plane of interest making this well suited for the functional approach. Nevertheless the functional binning approach enhances the statistic for the jet and demonstrates the applicability of the method to real-world data.

In addition to the one-point statistics shown we now also evaluate two-point velocity correlation functions. Figure 5 shows such an evaluation for the synthetic data-set and compares the usual midpoint method with a 


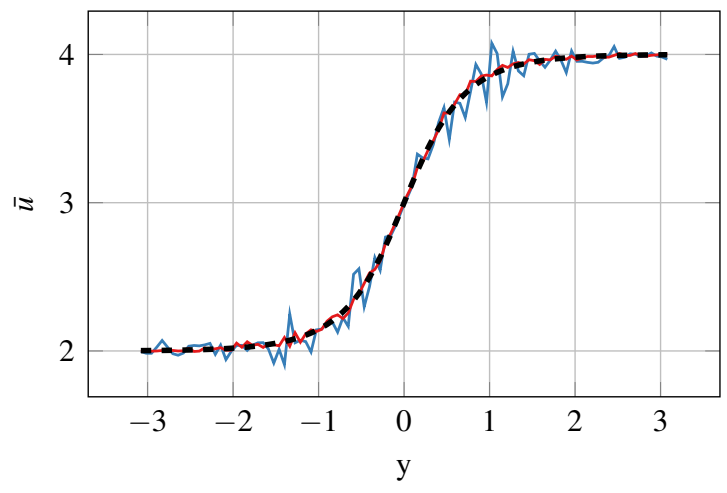

(a) Mean velocity value from synthetic experiment utilizing only 2000 images.

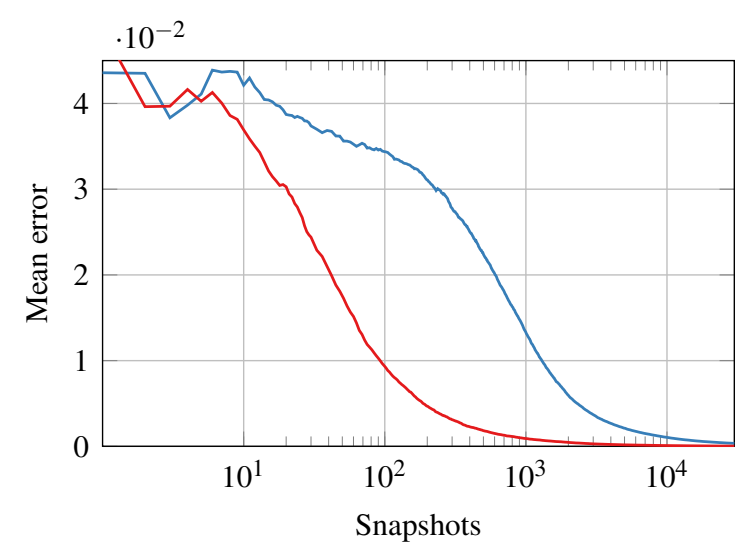

(b) Convergence behavior of the error of the mean velocity

Figure 3: Comparison of the functional to midpoint binning approach for the synthetic experiment. -Midpoint; —-Functional; - - - -Truth. Figures adapted from Godbersen and Schröder (2020)

function based approach. Similar qualitative improvements as with the one-point statistics are apparent. The features are smoother and the inflow region is filled as information along the entirety of the track is utilized. Both effect mirror those within the mean velocity field for both methods as shown in detailed one-point statistic evaluations in Godbersen and Schröder (2020).

\section{Further uses of uncertainty information}

So far examples of one-point statistics and two-point correlations were presented. But a functional view including uncertainty quantification is also beneficial for more complex two-point statistics as well. This approach can be very attractive for all statistics where relations between pairs of particles or triads have to be considered, for example the calculation of velocity gradients or velocity differences for structure functions. These calculations pose the problem of finding suitable pairs for these comparisons based on criterions such as a close enough distance. Instead of just considering the midpoint, we now have the entire length of the track segment to find eligible comparison points. A systematic approach for selection of comparison points is now also facilitated through the use of uncertainty quantification by propagating the particle positioning error. This is even more important here as the use of velocity or position differences for small position or velocity differences relies less directly on the absolute error but rather the relative error. Instead of just estimating a rough rule of thumb for the closest feasible minimum distance based of the position accuracy we can derive a direct functional expression for the respective relative errors along the length of the tracks by use of error propagation. This directly informs which regions in time along the track pair are unsuited for such comparisons. By prescribing some criterions for the admissible errors, such as some maximum value for the relative errors, we can directly determine permissible regions for calculating comparisons. Additionally, we can utilize this knowledge to help calculate an optimal point for calculating the comparison value. Typically, there is a desired criterion for calculating such a comparison value that based on the desired statistic. For a velocity gradient the particles should be as close together as possible in order to reduce truncation error in the velocity difference quotient used for the calculation. Such a goal can now be combined with the constraints imposed by the error analysis to identify the optimal point along the track pair for such a calculation.

To illustrate this approach, we present an example on a synthetic track pair in two dimensions. These two tracks were randomly generated in the box $[0,1] \times[0,1]$ and do not aim to represent an actual experiment but to provide an interesting illustration for the approach. In this example the velocity difference quotient is the targeted value for selecting an optimal point:

$$
\mathrm{vdq}=\frac{v_{2}-v_{1}}{p_{2}-p_{1}}
$$

as this allows to showcase multiple relative error values for distance and velocity difference. We now desire the optimal point along the track to evaluate this quotient, meaning the optimal value for the time $t$. Optimal in 


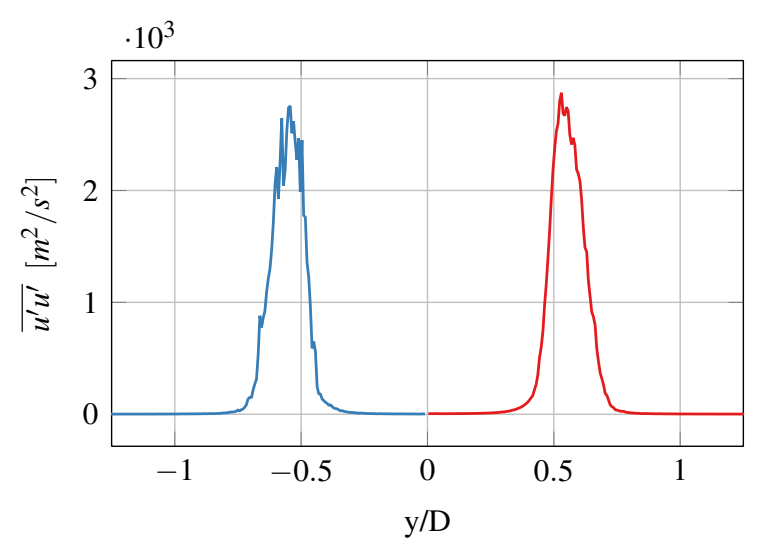

(a) Using only 1000 snapshots

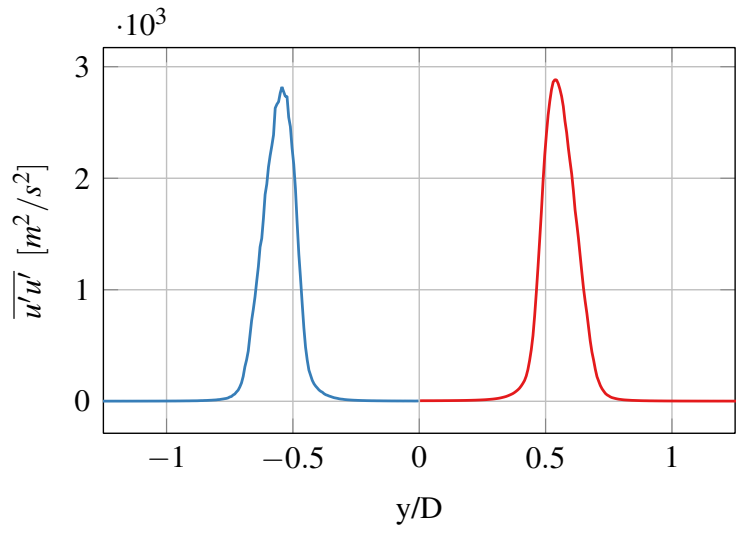

(b) Using 30000 snapshots

Figure 4: Comparison of the functional to midpoint binning approach for the real-word data. Axial Reynolds stress profile located ca. 1.5 nozzle diameters donwstream. Utilizing $3 \mathrm{px}$ bin size in axial and radial direction. -Midpoint; — Functional.
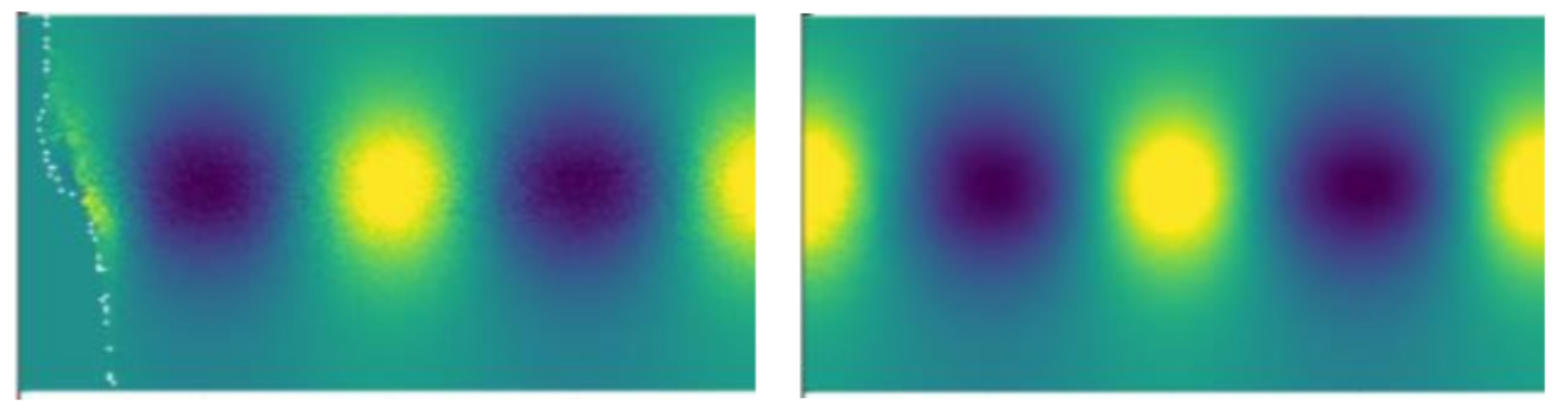

Figure 5: Qualitative comparison of two-point correlation results for the midpoint approach (left) and the functional approach (right)

this case means the closest point between the tracks that still conforms to some value of permitted maximum relative error. For illustration the two relative errors for distance and velocity difference are considered separately here but could also be joined into one single common error expression. Propagating the particle position error through the track building process and the further calculations as defined in (4) provides the relative errors as continuously defined functions within the track segment as shown in figure 6 . We use a software package to provide automatic propagation of uncertainty trough these functions (Giordano, 2016) allowing for easy generation of these error functions independent of the complexity of the input functions. It would generally still be possible to manually derive the functions if a mathematical expression is desired for further study. As visible in the figure the relative errors vary quite strongly for the tracks considered here. It is easy to identify regions were either one of the errors is significantly increased. As expected the relative difference error is higher the closer the two tracks approach each other. But also, the relative velocity difference error changes quite dynamically along the track showing the value of having such a direct continuous varying error value available. As mentioned earlier, only the particle positioning error was the required input for these results whereas all further influence of the processing and derivation of variables is directly captured by error propagation with no further input needed.

The resulting error functions can now be utilized to identify an optimal point for the calculation in (4). We minimize the distance between the tracks with a maximum constraint on the two relative errors resulting the in optimal point shown in figure 6. The cost function for the optimization could easily be extend to include additional concerns, such as penalizing close contact for the four pulse times where the particles are imaged. This provides the additional knowledge that close contact during those times could result in overlapping particle images. Currently we utilize a Simulated Annealing algorithm implemented in Mogensen and Riseth (2018) and manually include the constraints into the cost function using logarithmic 

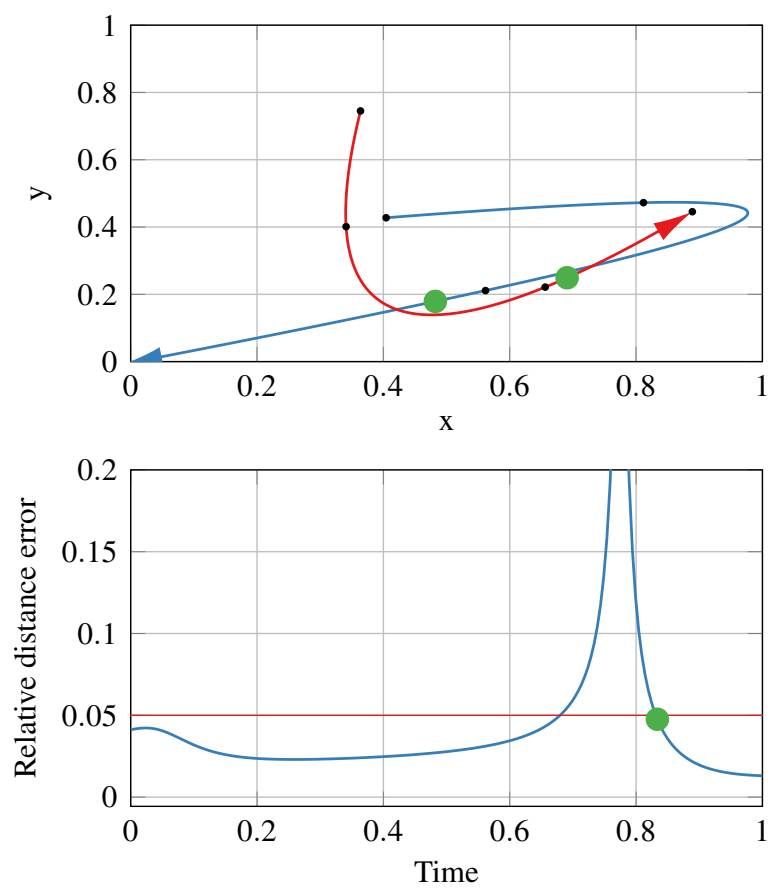
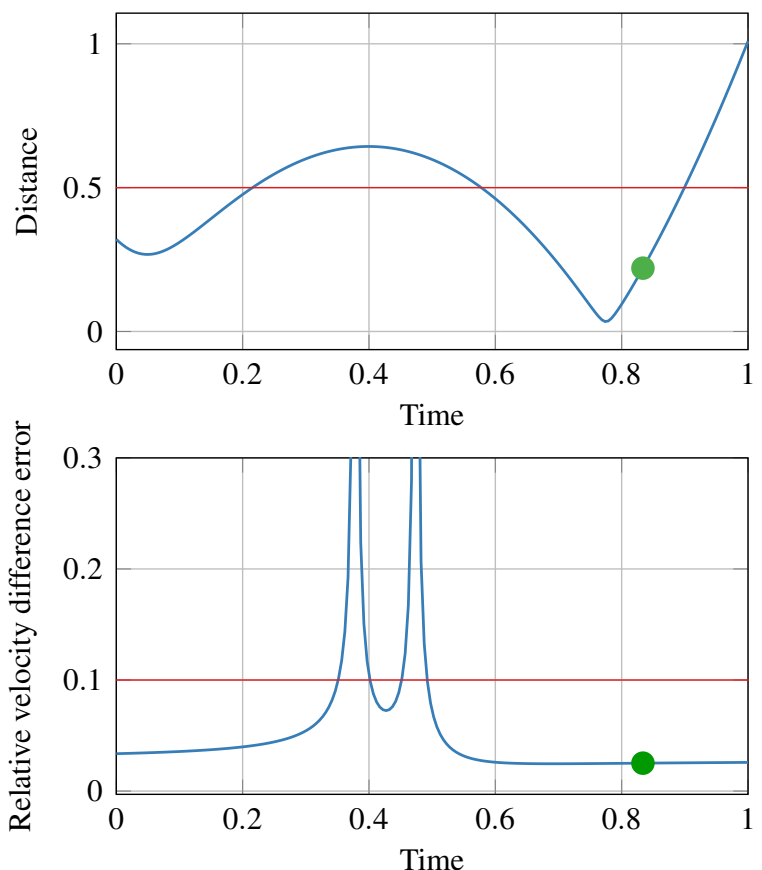

Figure 6: Error analysis for the calculation of the velocity difference quotient for two tracks with calculated optimal point in green. The red horizontal lines signify the selected upper constraints for the optimizer

barrier functions. The choice of simulated annealing was motivated by the desire to avoid local minima, other algorithms might be better suited for this application. Further work in this area will include a more systematic study of optimization algorithms, including ones natively supporting constraints.

\section{Individual uncertainty}

In the evaluation shown so far, only one global common particle position error value is used for all particles of the experiment. This already provides a diverse set of uncertainty in calculated values for tracks as these common errors are then individually propagated for each calculated variable leading to possibly quite complex interactions as seen in figure 6. Still one expects that there is some variation in the errors associated with individual particles due to different effects in the STB processing. A particle that is far away from any other particles in all camera images is very easy of the STB algorithm to generate good position for resulting in a lower than average positioning error for that particle. On the other hand, a particle that is very close to several other particles on the images might see a slightly higher position error due to overlap in the particles making reconstruction more challenging. As the camera images and positions of particles are known, it should be feasible to model this effect at least to some degree. A series of other effects can also be imagined such as distortion of images in some regions due to measurement windows, optical effects close to the edges of the camera lenses, illumination differences closer to the edges of the volume, etc. Some of these have probably have a minimal effect on the accuracy but most of the mentioned possible influences also have in common that it might be feasible to model them, even if just roughly. Many rely on particle locations with respect to the measurement volume or the camera image, information which is available from the measurement data.

While all examples shown so far only utilize one common particle position error value this is not due to a limitation of the method as nowhere in the derivation was this assumed. The method as currently implemented already allows for such individual values. An illustration of the effect of individual particle positioning errors for each of the particles within a track is provided in figure $7(\mathrm{a})$. The general V-shape stays the same since this is a result of the polynomial fit, however the location of minimal error shifts to different time points and the value of the errors changes. This information can then be propagated in the functional binning approach by utilizing individual track weights based on these error functions. The effect of this individual consideration on the resulting statistics will largely depend on the amount of variation in error between the tracks. For 


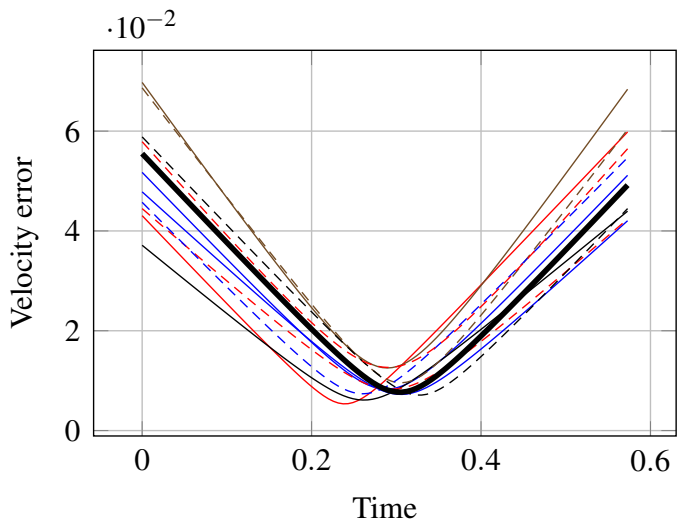

(a) Calculated velocity error curves for randomized individual particle positioning errors. Each line is based on a random draw of individual position error values for the four particles. Shown in thick black is the curve using identical particle error values.

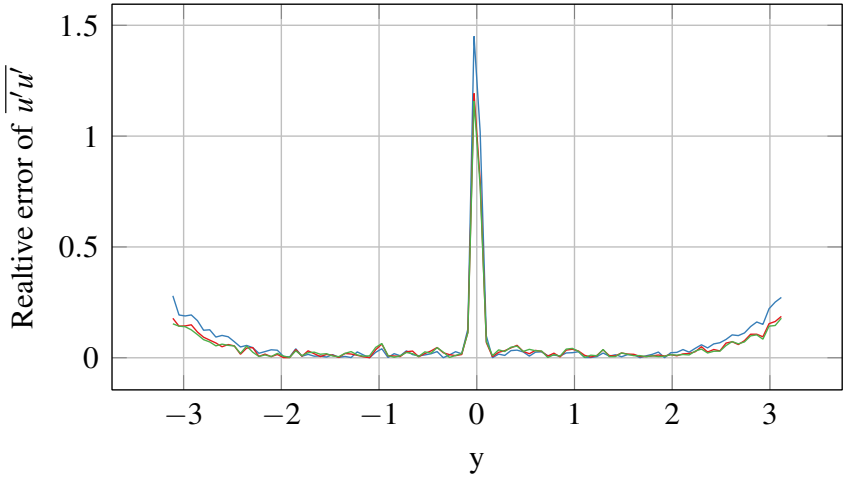

(b) Relative error to ground truth for the three different evaluations showing very similar values for the class based variant and the individual variant. Both show reduced error in some regions compared to the variant with a fixed global particle positioning error. G_ Global; -Individual; Class based.

Figure 7: The individual particle positioning error values allow for more detailed uncertainty knowledge.

experiments where there is little variation in error between the tracks only minimal difference to the approach using a global mean particle position error value would be expected.

To provide some insight into the effect of individual track weights we extend the synthetic experiment shown prior by such an individual error value. As the particle positioning error there is simply prescribed by adding Gaussian noise to the calculated particle positions, this switch simply requires modifying this noise source. Instead of a random draw from a normal distribution with a fixed standard deviation for all tracks, we now draw vary this standard deviation for each particle and communicate this value together with the position result. This setup results in particles where for high reported error value it is likely that a larger amount of noise has been added than for a particle with a low reported error value. The approach was selected to avoid reporting the exact error value added to each particle, as such knowledge would never be possible to archive in a real experiment. With the such prepared error information the synthetic data is now processed using Functional Binning with individual track weight functions and the such calculated Reynolds stresses then compared to the known ground truth data in a profile across the domain. As shown in the relative error plot in 7(b) there is an improvement when compared to the Functional Binning using a global mean particle positioning error. The regions where the improvement is visible are regions of lower absolute value of the analyzed Reynolds stress and therefore more sensitive for the relative error value. A second different evaluation is included in this comparison where instead of directly utilizing the error value reported by the synthetic experiment we pretend that instead we only know for each particle a membership to one of three quality groups. One group containing particles with above average error, one for particles of about average error value, and one for those with a below than average error value. The motivation behind this is that such a rough classification might be more readily achievable for real measurement data. As seen in the figure this simpler approach already provides much of the improvement archived by the approach utilizing more detailed error information.

With these results in mind we are currently working on a machine learning model to provide such error estimation classification for real world measurements in order to apply Functional Binning with such individual track weight functions. The model utilizes information from the shaking step of STB processing such as gradients and hessians from the optimization procedure This is a core portion of the processing where all the information about the camera images and particle positions comes together providing a wealth of condensed information to the learning process. Originally this model was designed to provide a better filter for ghost particles than the currently used conventional filter and has shown some success on the test datasets provided by the LPT-Challenge organizers (Sciacchitano et al., 2021). Extending the approach used for the machine learning model to provide a rough certainty classification instead seems feasible as the task is closely related, however this needs to be pursued further. 


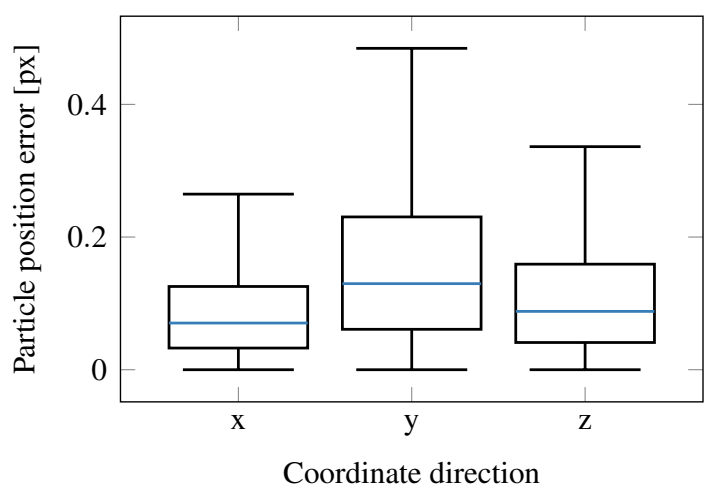

(a) Particle position error estimation for multi-pulse STB based on measurement data. The separation into direction would also allow for utilizing individual error values for each of the coordinate directions

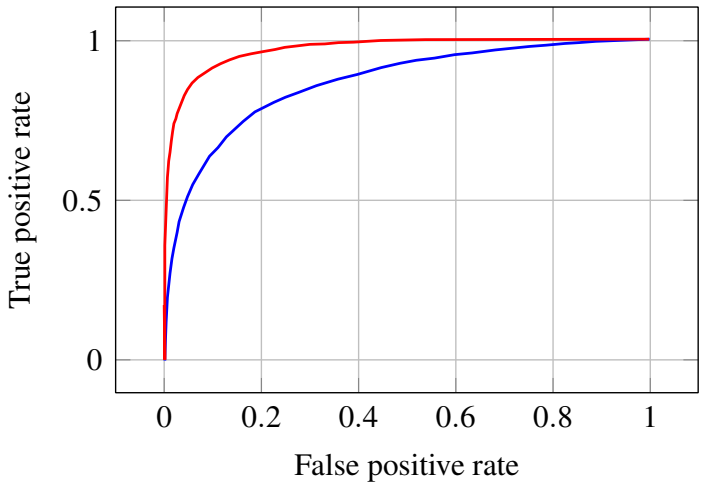

(b) Performance comparison between the original filter and the machine learning model for identifying ghost particles in a synthetic measurement. Conventional; - Machine learning model .

Figure 8: Statistical evaluation on the measurement data allow for a posteriori uncertainty estimation to obtain a global particle positioning error. Modifications to an existing Machine Learning model could provide a path towards particle individual position uncertainty estimation.

\section{Conclusion}

We have shown several applications of uncertainty quantification in Functional Binning and related approaches on Lagrangian Particle Tracking data and illustrated the improvements over conventional approaches. Functional Binning uses uncertainty information of the measurement data as a core feature of the method to improve the calculation of statistics an provides a readily available way to introduce such information into the calculation of the various statistics. The determination of one-point statistics was demonstrated not just on the existing evaluation on synthetic data but for the first time on an actual multi-pulse STB dataset. While the lack of ground truth data does not permit a quantitative evaluation, the qualitative improvement visible when processing only a small subset of the data where the unconverged noisy profiles make any improvements immediately apparent is evident. This shows that an improvement in convergence speed is facilitated trough the functional binning approach not just for the synthetic experiment considered previously but also in a real-world multi-pulse STB measurement. The evaluation of two-point correlations is a natural extension of the one-point statistics shown so far, and the convergence improvement provided by the Functional Binning approach was demonstrated.

We strengthen the link to the initial uncertainty analyses described in the publications relating to the two STB variants by emphasizing the reliance on an a posteriori uncertainty quantification of the particle positioning error provided by these as a basis on which all further error processing in functional binning is built on by propagation this error onwards. This also forms an interface for other LPT methods to utilize functional binning with no need to adapt its internals. As long as another method is capable of providing such particle positioning error these can then be propagated into the Functional Binning procedure.

Apart from the already more matured functional binning statistics mentioned above we also demonstrated an additional application in the form of the optimal point calculation for track-to-track comparisons. The advantage of error propagation was easy to see in this example and an optimal point was successfully determined. However, this approach still needs additional work as just a single optimal point calculation was demonstrated. The binning procedure surrounding this in order to be able to calculate statistics still needs to be completed so no actual statistical evaluations could be shown yet. Still the core idea behind this approach was successfully demonstrated. The use and estimation of particle individual error values is similarly a topic were initial successes were presented but additional work is needed to fully utilize these concepts. Conceptionally the Functional Binning approach is already prepared to utilize individual particle positioning errors for tracks and the presented synthetic experiment evaluation showed that there is value in such an extension. However, a more systematic evaluation of such an experiment is needed to gain a better understanding of the benefits depending on the amount of error variation in the measurement. To truly utilize this capability with real world measurement data it is necessary to extract such individual error estimation from the measurement data. We have described why this should be feasible with the available measurement 
data, especially with the reduction to just a classification into a limited number of groups. For initial work on a machine learning model to provide such classification we were able to present progress in a closely related task, putting this capability into reach but requiring further work.

The presented evaluations and ideas highlight the large amount of opportunities that uncertainty quantification approaches provide in the improvement of one- and two-point statistics, especially in the context of Functional Binning which provides a readily available path to incorporate this uncertainty information into the calculation of statistics of LPT data. It is also apparent that while some approaches are relatively mature and ready for application to measurements, there are still many interesting ideas and extensions left to explore and further develop.

\section{Acknowledgements}

The authors gratefully acknowledge the Gauss Centre for Supercomputing e.V. (www.gauss-centre.eu) for funding this project by providing computing time on the GCS Supercomputer SuperMUC at Leibniz Supercomputing Centre (www.lrz.de).

The project leading to this contribution has received funding in the frame of the project HOMER from the European Union's Horizon 2020 research and innovation program under grant agreement No. 769237.

\section{References}

Agüí JC and Jiménez J (1987) On the performance of particle tracking. Journal of Fluid Mechanics 185:447-468

Agüera N, Cafiero G, Astarita T, and Discetti S (2016) Ensemble 3d PTV for high resolution turbulent statistics. Measurement Science and Technology 27:124011

Bhattacharya S and Vlachos PP (2020) Volumetric particle tracking velocimetry (PTV) uncertainty quantification. Experiments in Fluids 61:1-18

Bogaert I (2014) Iteration-free computation of gauss-legendre quadrature nodes and weights. SIAM Journal on Scientific Computing 36:A1008-A1026

Gesemann S, Huhn F, Schanz D, and Schröder A (2016) From noisy particle tracks to velocity, acceleration and pressure fields using B-splines and penalties. in 18th international symposium on applications of laser and imaging techniques to fluid mechanics, Lisbon, Portugal. pages 4-7

Gesemann et al (2021) Trackfit: Uncertainty quantification, optimal filtering and interpolation of tracks for time-resolved lagrangian particle tracking. in 14th International Symposium on Particle Image Velocimetry

Giordano M (2016) Uncertainty propagation with functionally correlated quantities. arXiv e-prints arXiv:1610.08716

Godbersen P, Manovski P, Novara M, Schanz D, Geisler R, Mohan NKD, and Schröder A (2019) Flow field analysis of subsonic jets at Mach 0.5 and 0.84 using 3D Multi Pulse STB. in Conference Proceedings of the 13th International Symposium on Particle Image Velocimetry. 202. pages 545-554

Godbersen P and Schröder A (2020) Functional binning: improving convergence of eulerian statistics from lagrangian particle tracking. Measurement Science and Technology 31:095304

Hammond A and Meng H (2021) Particle radial distribution function and relative velocity measurement in turbulence at small particle-pair separations. Journal of Fluid Mechanics 921:A16

Manovski P, Novara M, Mohan NKD, Geisler R, Schanz D, Agocs J, Godbersen P, and Schröder A (2021) 3D Lagrangian particle tracking of a subsonic jet using multi-pulse Shake-The-Box. Experimental Thermal and Fluid Science 123:110346

Mogensen PK and Riseth AN (2018) Optim: A mathematical optimization package for Julia. Journal of Open Source Software 3:615 
Novara M, Schanz D, Geisler R, Gesemann S, Voss C, and Schröder A (2019) Multi-exposed recordings for 3D Lagrangian particle tracking with Multi-Pulse Shake-The-Box. Experiments in Fluids 60:44

Novara M, Schanz D, Reuther N, Kähler CJ, and Schröder A (2016) Lagrangian 3d particle tracking in high-speed flows: Shake-The-Box for multi-pulse systems. Experiments in Fluids 57:128

Ouellette NT, Xu H, and Bodenschatz E (2006) A quantitative study of three-dimensional lagrangian particle tracking algorithms. Experiments in Fluids 40:301-313

Raiola M, Lopez-Nuñez E, Cafiero G, and Discetti S (2020) Adaptive ensemble PTV. Measurement Science and Technology 31:085301

Schanz D, Gesemann S, and Schröder A (2016) Shake-The-Box: Lagrangian particle tracking at high particle image densities. Experiments in Fluids 57:70

Sciacchitano A, Leclaire B, and Schröder A (2021) Main results of the first lagrangian particle tracking challenge. in 14th International Symposium on Particle Image Velocimetry

Van Gent P, Michaelis D, Van Oudheusden B, Weiss PÉ, de Kat R, Laskari A, Jeon YJ, David L, Schanz D, Huhn F et al. (2017) Comparative assessment of pressure field reconstructions from particle image velocimetry measurements and lagrangian particle tracking. Experiments in Fluids 58:33

Wieneke B (2008) Volume self-calibration for 3d particle image velocimetry. Experiments in fluids 45:549556 